\title{
CORRELAÇÕES GENÉTICAS E ANÁLISE DE TRILHA PARA CARACTERES DE FRUTO DA PALMEIRA JUÇARA ${ }^{1}$
}

\author{
TIAGO DE SOUZA MARÇAL ${ }^{2}$, ADÉSIO FERREIRA ${ }^{3}$, \\ WAGNER BASTOS DOS SANTOS OLIVEIRA ${ }^{4}$, JOSÉ HENRIQUE SOLER GUILHEN ${ }^{2}$, \\ MARCIA FLORES DA SILVA FERREIRA ${ }^{3}$
}

RESUMO - A palmeira Euterpe edulis é uma espécie nativa da Mata Atlântica e atualmente se encontra na lista das espécies ameaçadas de extinção. Uma alternativa para retirá-la desta lista seria o estímulo para o plantio comercial, focando o manejo dos frutos, que recebem a classificação de "superfruta" pelas suas propriedades químicas e nutricionais. Entretanto, uma etapa de extrema importância que precede a seleção de genótipos superiores é o estudo das associações entre as variáveis, pois permite traçar estratégias de seleção alternativas para maximizar os ganhos. O presente trabalho teve por objetivos estimar as correlações genéticas pelo procedimento REML/BLUP e os efeitos diretos e indiretos sobre a massa dos frutos, por meio da análise de trilha, para seis caracteres de frutos de 198 acessos de E. edulis. Foram analisados frutos de 198 genótipos de juçara coletados em 20 fragmentos florestais na região Sul e Caparaó do Estado do Espírito Santo. De cada genótipo, avaliaram-se 25 frutos para as características: diâmetro longitudinal e equatorial do fruto e da semente; e massa do fruto e da semente. Os dados obtidos foram utilizados para a estimativa das correlações genéticas através do método de máxima verossimilhança restrita e melhor predição linear não viesada (REML/BLUP). Posteriormente, as correlações genéticas entre as variáveis de fruto foram submetidas à análise de trilha. Os seis caracteres de fruto estudados apresentam associação genética positiva com magnitude superior a 0,71 pelo procedimento REML/BLUP. O diâmetro longitudinal do fruto e a massa das sementes possuem maior efeito direto sobre a massa dos frutos, o que as torna mais indicadas para aumentar as chances de sucesso na seleção de genótipos de juçara com frutos maiores. As características diâmetro longitudinal do fruto e a massa das sementes são as principais determinantes das variações na massa dos frutos.

Termos para indexação: Euterpe edulis, Pré-melhoramento, Seleção de Caracteres, Análise Biométrica.

\section{GENETIC CORRELATIONS AND PATH ANALYSIS FOR FRUIT CHARACTERS OF JUÇARA PALM TREE}

\begin{abstract}
The palm tree Euterpe edulis is a native species from Mata Atlântica and it is nowadays found in the list of species which are threatened by extinction. One option to withdraw it from this list would be the stimulation of the commercial planting aiming the management of fruits which receive the "super fruit" classification by their chemical and nutritional properties. However, one stage of extreme importance that precedes the superior genotypes selection is the study of associations among the variables, because it allows delineating alternate selection strategies to maximize the gains. The present study had as objective to estimate the genetic correlations by the REML/BLUP procedure and the direct and indirect effects upon fruit mass by path analysis for six fruit characters of 198 accessions of $E$. edulis. Fruits of 198 Juçara genotypes collected in 20 forestry fragments in the south and Caparaó region of the state of Espírito Santo were analyzed. From each genotype 25 fruits were evaluated for the characteristics: longitudinal and equatorial fruit and seed diameter; fruit and seed mass. The obtained data were used for genetic correlations estimative through the method of restricted maximum likelihood and better unbiased linear prediction (REM/BLUP). Subsequently, the genetic correlations among the fruit variables were submitted to path analysis. The six fruit characters studied show positive genetic association with magnitude higher than 0.71 by the REML/BLUP procedure. The longitudinal diameter of fruit and seed mass have greater direct effect on fruit weight, which makes them most indicated to increase the chances of success in the Juçara genotypes selection with larger fruit . The characteristics longitudinal diameter of fruit and the seed mass are the main determinants of changes in the mass of fruit.

Index terms: Euterpe edulis, Pre-improvement, Characters Selection, Biometric Analysis.

1(Trabalho 163-14). Recebido em: 09-05-2014. Aceito para publicação em: 09-04-2015.

${ }^{2}$ Eng. Agr., Universidade Federal do Espírito Santo, Departamento de Produção Vegetal, Campus Alegre, 29500-000, Alegre - ES, Brasil. E-mail: jhguilhen@gmail.com; tiagosmaragronomia@gmail.com

${ }^{3}$ Doutor (a) Professor (a) Adjunto, Universidade Federal do Espírito Santo, Departamento de Produção Vegetal, Campus Alegre, 29500000, Alegre - ES. E-mail: adesioferreira@gmail.com; mfloressf@gmail.com

${ }^{4}$ Mestre em Produção Vegetal, Universidade Federal do Espírito Santo, Departamento de Produção Vegetal, Campus Alegre, 29500000, Alegre - ES. E-mail: wobastos@yahoo.com.br
\end{abstract}




\section{INTRODUÇÃO}

A família Arecaceae possui representantes por toda a região tropical e subtropical do globo, sendo considerada uma importante fonte de recursos para a sobrevivência de diferentes comunidades (ZAMBRANA et al., 2007; BARFOD et al., 2011). Dentro desta família, o gênero Euterpe reúne 28 espécies (JARDIM, 2005), e destas, sete são distribuídas na América do Sul e Central, das quais seis são encontradas no Brasil: E. edulis, E. caatinga, E. oleracea, E. longebracteata, E. precatoria e E. espiritosantensis (PINATUD et al., 2008).

Duas espécies são destaque neste gênero por apresentar grande importância econômica e cultural (GATTI et al., 2011): a juçara (E. edulis) e o açaizeiro (. oleraceae), que apresentam potencial de produção de frutos (FARIAS NETO et al., 2007; OLIVEIRA; FARIAS NETO, 2008; PALUDO et al., 2012) com elevados teores de compostos funcionais e antioxidantes na polpa (COISSON et al., 2005), conferindo às mesmas a classificação de "superfruta" (SANTOS et al., 2014).

A juçara, que é uma palmeira nativa da Mata Atlântica, atualmente está incluída na lista das espécies ameaçadas de extinção (BRANCALION et al., 2012; SGROTT et al., 2012). Isso se deve ao fato, principalmente, da prática predatória do palmito (BARROSO et al., 2010; BATAGIN-PIOTTO et al., 2012), associada ao longo período juvenil da espécie, que dura de 6 a 9 anos (CEMBRANELI et al., 2009).

O manejo apropriado dos recursos genéticos, a conservação in situ, a delimitação de áreas de proteção, entre outros fatores, podem evitar o risco de extinção de uma espécie (SHIMIZU, 2007). Para palmiteiro-juçara, o estímulo para a utilização comercial de seus frutos é uma ação que pode contribuir para a retirada da juçara da lista brasileira de espécies ameaçadas de extinção; entretanto, para a espécie ser utilizada comercialmente, há a necessidade de se alcançar maior produtividade, selecionando genótipos mais adaptados e produtivos.

Uma etapa de extrema importância que precede a seleção é o estudo das associações entre as variáveis, pois em função da complexidade ou da dificuldade operacional de fenotipagem de alguns caracteres, pode-se lançar mão da seleção indireta (CRUZ et al., 2012). Além disso, o conhecimento das correlações permite traçar estratégias de seleção alternativas para maximizar os ganhos (TEIXEIRA et al., 2012).

O coeficiente de correlação estimado para duas variáveis pode estar recebendo a influência de outros caracteres (CRUZ et al., 2012). Nesse sentido, a análise de trilha ("path analysis"), proposta por Wright (1921), permite estudar mais detalhadamente as relações e as inter-relações existentes entre um grupo de características de interesse.

Considerando a importância da análise de trilha no estudo das correlações, diversos autores têm empregado este procedimento para gerar informações em programas de melhoramento de fruteiras, como maracujazeiro-amarelo (NEGREIROS et al., 2007), pereira (GALARÇA et al.,2010), mamoeiro (OLIVEIRA et al., 2010) e açaizeiro (TEIXEIRA et al., 2012).

O presente trabalho teve por objetivo estimar as correlações genéticas pelo procedimento REML/ BLUP e os efeitos diretos e indiretos sobre a massa dos frutos, por meio da análise de trilha, para seis caracteres de frutos de 198 acessos de E. edulis.

\section{MATERIAL E MÉTODOS}

Foram analisados frutos de 198 genótipos de juçara coletados em 20 fragmentos florestais, na microrregião Sul (munícipios - Muqui, Mimoso e Jerônimo Monteiro) e Caparaó (munícipios - Alegre, Guaçuí e Ibitirama), do Estado do Espírito Santo. Nos fragmentos, foram coletados frutos de dez genótipos, com exceção de um dos fragmentos do munícipio de Guaçuí, no qual foram colhidos frutos de oito genótipos.

De cada genótipo, foram avaliados 25 frutos para as características: diâmetro longitudinal e equatorial do fruto e da semente (DLF, DLS, DEF e DES), em milímetros (mm), com auxílio de um paquímetro digital de $0,01 \mathrm{~mm}$ de precisão; a massa do fruto e da semente (MF e MS), em gramas(g), com ajuda de balança eletrônica de $0,01 \mathrm{~g}$ de precisão.

A análise dos dados foi realizada pelo método de máxima verossimilhança restrita e melhor predição linear não viesada (REML/BLUP), utilizando o modelo básico de repetibilidade que assume ausência de delineamento, podendo ser escrito na forma matricial, através da equação seguinte (RESENDE, 2007):

$$
y=X m+Z p+\varepsilon
$$

em que:

y: é o vetor da variável a ser analisada;

$\mathrm{m}$ : é o vetor de efeitos de medições assumidos como fixos e somados à média geral;

p: é o vetor de efeitos fenotípicos permanentes assumidos como aleatórios;

$\varepsilon$ : é o vetor de erros aleatórios; 
$X$ : a matriz de incidência para os efeitos fixos;

$\mathrm{Z}$ : é a matriz de incidência para os efeitos fenotípicos permanentes.

Após a obtenção das médias corrigidas pelo procedimento BLUP, foram estimadas as correlações genéticas entre as variáveis analisadas (RESENDE, 2007), através do seguinte estimador:

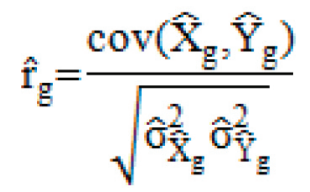

em que:

$\hat{\mathbf{1}}_{\text {g: Estimativa da correlação genética entre a }}$ variável X e Y;

$\widehat{\mathrm{X}}_{\mathrm{g}}$ : Estimativa do valor genotípico para a variável $\mathrm{X}$;

$\widehat{\mathrm{Y}}_{\mathrm{g}}$ : Estimativa do valor genotípico para a variável Y;

$\hat{\sigma}_{\mathrm{X}_{\mathrm{g}}}^{2}$ : Estimativa da variância genética do valor genotípico estimado para a variável $\mathrm{X}$;

$\widehat{\sigma}_{\widehat{Y}}^{2}$ : Estimativa da variância genética do valor genotípico estimado para a variável Y.

Posteriormente, as correlações genéticas serviram como base para o diagnóstico de multicolinearidade, para verificar se a condição de multicolinearidade fraca $(\mathrm{NC}<100)$, proposta por Montgomery e Peck (1981), havia sido atendida no intuito de se evitar superestimativas dos coeficientes de trilha (CRUZ et al., 2012).

A análise de trilha foi realizada com base na matriz de correlações genéticas, utilizando um estimador de mínimos quadrados (CRUZ et al., 2012).

$$
\left[\begin{array}{ccccc}
1 & r_{12} & r_{13} & \ldots & r_{1 j} \\
r_{21} & 1 & r_{23} & \ldots & r_{2 j} \\
r_{31} & r_{32} & 1 & \cdots & r_{3 j} \\
\vdots & \vdots & \vdots & \ddots & \vdots \\
r_{11} & r_{i 2} & r_{i 3} & \cdots & 1
\end{array}\right]\left[\begin{array}{c}
p_{01} \\
p_{02} \\
p_{03} \\
\vdots \\
p_{01}
\end{array}\right]=\left[\begin{array}{c}
r_{01} \\
r_{02} \\
r_{03} \\
\vdots \\
r_{01}
\end{array}\right]
$$

em que:

X'X : Matriz de correlações genéticas entre variáveis explicativas;

$\beta$ : Coeficiente da análise de trilha;

X’y : Correções genéticas da variável de efeito principal com as variáveis explicativas.

As análises REML/BLUP, correlações genéticas e de trilha foram realizadas no programa R (TEM, 2013).

\section{RESULTADOS E DISCUSSÃO}

As correlações genéticas estimadas foram altas e positivas entre todas as características, sendo superiores a 0,70; destacando-se MF com DEF $(0,98)$ e MS com DES $(0,93)$ (Tabela 1). Segundo Cruz et al. (2012), a existência de associação genética positiva entre dois caracteres implica que a alteração em um deles pode provocar mudança, no mesmo sentido, no outro.

A correlação obtida diretamente dos dados colhidos é a fenotípica, cujas causas são genéticas e ambientais; entretanto, somente a genética é de natureza herdável. Portanto, deve-se buscar estimar a correlação genética em programas de melhoramento (CRUZ et al., 2012).

Além disso, as correlações genéticas podem ser muito úteis quando existem caracteres de baixa herdabilidade e/ou de difícil mensuração, pois nestas condições pode-se praticar a seleção indireta, desde que exista causa e efeito para as correlações estudadas (FALCONER, 1987; CRUZ et al., 2012).

Como todas as correlações foram positivas com MF, a seleção simultânea pode promover ganhos no peso de frutos de forma mais eficiente, pois, segundo Falconer e Mackay (1996), ao selecionar os caracteres que contribuem positivamente para o caráter de interesse, faz-se o uso da correlação de forma mais efetiva. Valores positivos para a correlação genética entre caracteres de fruto também foram encontrados por Teixeira et al. (2012) para açaizeiro.

O diagnóstico de multicolinearidade realizado com base nas correlações genéticas (Tabela 1) revelou o número de condições (NC) de 364,73, o que condiciona multicolinearidade moderada a forte (MONTGOMERY; PECK, 1981); portanto, poderia trazer prejuízo para a análise de trilha (COIMBRA et al., 2005).

A multicolinearidade é um fenômeno que ocorre quando as observações amostrais das variáveis explicativas, ou suas combinações lineares, são correlacionadas (MATSUO, 1986; FERRARI, 1989).

Verificado o problema da multicolinearidade, procedeu-se ao descarte de variáveis para que o $\mathrm{NC}$ se tornasse inferior a cem, atingindo assim a condição de multicolinearidade fraca (MONTGOMERY; PECK, 1981). A variável DEF foi a candidata ao descarte, e 
após sua retirada, o NC baixou para 83,76 e, então, procedeu-se às análises de trilha.

Atendido o critério de multicolinearidade, realizou-se a análise de trilha para tentar entender as inter-relações das variáveis DLF, DLS, DES, MS e MF, sendo MF considerada a variável principal, e as demais, variáveis dependentes.

Com esta análise, buscou-se verificar se a alta magnitude das correlações genéticas das variáveis básicas com variável principal (Tabela 2) se deve à contribuição indireta de uma ou mais das variáveis básicas. Segundo Cruz et al. (2012), a interpretação com base na magnitude de uma correlação pode resultar em equívocos no processo de seleção, pois a correlação elevada pode ser resultado de uma contribuição indireta de uma ou mais características do grupo de caracteres avaliados.

As características que apresentaram os maiores efeitos diretos sobre MF foram: $\operatorname{DLF}(0,649)$ e MS (0,397), sendo os efeitos diretos de DLS $(-0,081)$ e DES $(0,042)$ quase nulos. Além disso, as variáveis DLF e MS também apresentaram os maiores efeitos indiretos sobre as demais (Tabela $2)$. Estes fatos mostram que estes dois caracteres têm de ser considerados no melhoramento E. edulis para ganhos de massa do fruto.

Segundo Bhagavan e Nair (1989) e Sindhumole e Ibrahim (2002), as características que apresentam superioridade sobre a variável principal, em relação às demais, são de grande utilidade para a seleção de plantas, pois pode-se praticar a seleção indireta (CRUZ et al., 2012). Segundo Montardo et al. (2003), trabalhando com rendimento de sementes de trevo-vermelho (Trifolium pratense L.), a seleção indireta por caracteres relacionados a uma variável quantitativa pode ser mais eficiente em termos de ganho de seleção.

Vencovsky e Barriga (1992) enfatizaram, ainda, que o conhecimento das variáveis relacionadas com uma característica de interesse permite que não se perca o controle sobre o comportamento e o equilíbrio harmônico entre os componentes da variável de interesse, pois estes são os requisitos básicos para boa caracterização de uma cultivar.

Observou-se que os efeitos diretos de todas as variáveis sobre MF foram inferiores às correlações genéticas $\left(\mathrm{r}_{\mathrm{g}}\right)$ das mesmas com MF, indicando a existência de uma ou mais características, não consideradas neste estudo, que têm influência sobre a variável MF.

Este fato é reforçado por o coeficiente de determinação da análise de trilha $\left(\mathrm{R}^{2}\right)$ encontrado $(0,88)$ ter sido inferior a um. Desta forma, percebese que a variação da variável principal não foi totalmente explicada por este esquema causal (Tabela 2). Resultado similar foi encontrado por Amorim et al. (2008) em estudo de análise de trilha, tendo como efeito principal o rendimento de grãos de girassol.

TABELA 1 - Correlações genéticas $\left(\mathrm{r}_{\mathrm{g}}\right)$ entre as variáveis diâmetro longitudinal e equatorial do fruto e da semente (DLF, DEF, DLS e DES), e massa do fruto e da semente (MF e MS) em 198 acessos de Euterpe edulis.

\begin{tabular}{ccccccc}
\hline Variável & DLF $(\mathrm{mm})$ & DEF $(\mathrm{mm})$ & DLS $(\mathrm{mm})$ & DES $(\mathrm{mm})$ & MF $(\mathrm{g})$ & MS $(\mathrm{g})$ \\
\hline DLF & 1 & 0,86 & 0,82 & 0,71 & 0,90 & 0,72 \\
DEF & & 1 & 0,82 & 0,78 & 0,98 & 0,83 \\
DLS & & & 1 & 0,87 & 0,85 & 0,90 \\
DES & & & & 1 & 0,80 & 0,93 \\
MF & & & & & 1 & 0,83 \\
MS & & & & & & 1 \\
\hline
\end{tabular}


TABELA 2 - Estimativa de efeitos diretos e indiretos das variáveis dependentes: diâmetro longitudinal do fruto (DLF), diâmetro longitudinal da semente (DLS), diâmetro equatorial da semente (DLF) e massa da semente (MS) sobre a variável principal massa do fruto (MF) através da análise de trilha baseada nas correlações genéticas obtidas de 198 acessos de Euterpe edulis.

\begin{tabular}{ccc}
\hline Variáveis & Efeitos & Coeficiente de trilha \\
\hline \multirow{3}{*}{ DLF $(\mathrm{mm})$} & Efeito direto sobre MF & 0,649 \\
& Efeito indireto via DLS & $-0,067$ \\
& Efeito indireto via DES & 0,029 \\
& Efeito indireto via MS & 0,285 \\
& Total ( $\left.\mathrm{r}_{\mathrm{g}}\right)$ & 0,90 \\
\hline \multirow{3}{*}{ DLS $(\mathrm{mm})$} & Efeito direto sobre MF & $-0,081$ \\
& Efeito indireto via DLF & 0,532 \\
& Efeito indireto via DES & 0,036 \\
& Efeito indireto via MS & 0,358 \\
& Total ( $\left.\mathrm{r}_{\mathrm{g}}\right)$ & 0,85 \\
\hline \multirow{2}{*}{$\mathrm{DES}(\mathrm{mm})$} & Efeito direto sobre MF & 0,042 \\
& Efeito indireto via DLF & 0,459 \\
& Efeito indireto via DLS & $-0,071$ \\
& Efeito indireto via MS & 0,370 \\
& Total ( $\left.\mathrm{r}_{\mathrm{g}}\right)$ & 0,80 \\
\hline $\mathrm{R}{ }^{2}$ & Efeito direto sobre MF & 0,397 \\
$\mathrm{EVR}$ & Efeito indireto via DLF & 0,465 \\
$\mathrm{MS}(\mathrm{g})$ & Efeito indireto via DLS & $-0,073$ \\
& Efeito indireto via DES & 0,039 \\
& Total ( $\mathrm{r}_{\mathrm{g}}$ ) & 0,83 \\
\hline
\end{tabular}

$\mathrm{r}_{\mathrm{g}}$ - Correlação genética, $\mathrm{R}^{2}$ - coeficiente de determinação da análise de trilha e EVR - efeito da variável residual.

\section{CONCLUSÕES}

Os seis caracteres de fruto estudados apresentam associação genética positiva com magnitude superior a 0,71 pelo procedimento REML/BLUP.

O diâmetro longitudinal do fruto e a massa das sementes possuem maior efeito direto sobre a massa dos frutos, o que as torna mais indicadas para aumentar as chances de sucesso na seleção de genótipos de juçara com frutos maiores.

As características diâmetro longitudinal do fruto e a massa das sementes são as principais determinantes das variações na massa dos frutos.

\section{AGRADECIMENTOS}

Agradecemos à Fundação de Amparo e Pesquisa do Espírito Santo - FAPES, ao Conselho Nacional de Desenvolvimento Científico e Tecnológico - CNPq, e à Coordenação de Aperfeiçoamento de Pessoal de Nível Superior CAPES, pelo fomento financeiro.

\section{REFERÊNCIAS}

AMORIM, E. P.; RAMOS, N. P.; UNGARO, M. R. G; KIIL, T. A. M. Correlações e análise de trilha em girassol. Bragantia, Campinas, v. 67, n. 2, p. 307-316, 2008. 
BARFOD, A. S.; HAGEN, M; BORCHSENIUS, F. Twenty-five years of progress in understanding pollination mechanisms in palms (Arecaceae). Annals of Botany, London, v. 108, n. 8 p. 1503$1516,2011$.

BARROSO, R. M.; REIS, A.; HANAZAKI, N. Etnoecologia e etnobotânica da palmeira juçara (Euterpe edulis Martius) em comunidades quilombolas do Vale do Ribeira, São Paulo. Acta Botânica Brasílica, São Paulo, v. 24, n. 2, p. 518528, 2010.

BATAGIN-PIOTTO, K. D.; ALMEIDA, C. V.; PIOTTO, F.A.; ALMEIDA, M. Anatomical analysis of peach palm (Bactris gasipaes) leaves cultivated in vitro, ex vitro and in vivo. Brazilian Journal of Botany, São Paulo, v. 35 n. 1, p. 71-78, 2012.

BHAGAVAN, S.; NAIR, B.P. Vigour index as an additional parameter in identifying elite palms in arecanut. Journal of Plantation Crops, Kasaragod, v.16, p-389-394, 1989.

BRANCALION, P. H. S.; VIDAL, E; LAVORENTI, N. A.; BATISTA, J. L. F.; RODRIGUES, R. R. Soil-mediated effects on potential Euterpe edulis (Arecaceae) fruit and palm heart sustainable management in the Brazilian Atlantic Forest. Forest Ecology and Management, New York, v.284, p.78-85, 2012.

CEMBRANELI, F.; FISCH, S. T. V.; CARVALHO, C. P. Exploração sustentável da palmeira Euterpe edulis Martius no Bioma Mata Atlântica, Vale do Paraíba - SP. Revista Ceres, Viçosa, MG, v. 56, n. 3, p. 233-240, 2009.

COIMBRA, J. L. M.; BENIN, G.; VIEIRA, E. A; OliveIRA, A. C.; CARVAlHO, F. I. F; GUIDOLIM, A. F; SOARES, A. P. Conseqüências da multicolinearidade sobre a análise de trilha em canola. Ciência Rural, Santa Maria, v. 35, n. 2, p. 347-352, 2005.

COISSON, J. D.; TRAVAGLIA, F.; PIANA, G.; CAPASSO, M.; ARLORIO, M. Euterpe oleracea juice as a functional pigment for yogurt. Food Research International, New York, v. 38, p. 893-897, 2005.
CRUZ, C. D.; REGAZZI, A. J; CARNEIRO, P. C. S. Modelos biométricos aplicados ao melhoramento genético. 4.ed. Viçosa: UFV, 2012. 514p.

FALCONER, D. S. Introdução à genética quantitativa. Viçosa: Imprensa Universitária, 1987. 279p.

FALCONER, D. S.; MACKAY, T. F. C. Introduction to quantitative genetics. London: Longman Malaysia, 1996. 463 p.

FARIAS NETO, J. T. de.; RESENDE, M. D. V. de.; OLIVEIRA, M. do S. P. de.; SANTOS, N. S. A. dos.; CANUTO, E. L.; NOGUEIRA, O. L. N.; MULLER, A. A. Avaliação genética de progênies de polinização aberta de açaí (Euterpe oleracea) e estimativas de parâmetros genéticos. Cerne, Lavras, v. 13, n. 4, p. 376-383, 2007.

FERRARI, F. Estimadores viesados para modelos de regressão em presença de multicolinearidade. Piracicaba: USP-ESALQ, 1989. 127p.

GALARÇA, S. P.; LIMA, C. S. M.; SILVEIRA, G.; RUFATO, A. R. Correlação de pearson e análise de trilha identificando variáveis para caracterizar porta-enxerto de Pyrus communis L. Ciência e Agrotecnologia, Lavras, v. 34, n. 4, p. 860-869, 2010 .

GATTI, M. G.; CAMPANELLO, P. I.; GOLDSTEIN, G. Growth and leaf production in the tropical palm Euterpe edulis: Light conditions versus developmental constraints. Flora, New York, v. 206, p. 742-748, 2011.

JARDIM, M. A. G. Possibilidade do cultivo do açaizeiro (Euterpe oleracea Mart.) em áreas de capoeira como alternativa para agricultores do nordeste do Nordeste Paraense. Belém: Museu Paraense Emílio Goeldi/Conselho Nacional de Desenvolvimento Científico e Tecnológico, 2005. 35 p. (Relatório Técnico Científico)

MATSUO, T. O uso da regressão de cumeeira em experimentos agronômicos. Piracicaba: USPESALQ, 1986. 89p.

MONTARDO, D. P.; DALL'AGNOL, M.; CRUSIUS, A. F.; PAIM, N. R. Análise de trilha para rendimento de sementes em trevo vermelho (Trifolium pratense L.). Revista Brasileira de Zootecnia, Viçosa, MG, v. 32, n.5, p.1076-1082, 2003. 
MONTGOMERY, D.C.; PECK, E.A. Introduction to linear regression analysis. New York : John Wiley, 1981. 504p.

NEGREIROS, J. R. S.; ÁlVARES, V. S.; BRUCKNER, C. H.; MORGADO, M. A. D.; CRUZ, C. D. Relação entre características físicas e o rendimento de polpa de maracujá-amarelo. Revista Brasileira de Fruticultura, Jaboticabal, v. 29, n. 3, p. 546-549, 2007.

OLIVEIRA, E. J.; LIMA, D. S. de.; LUCENA, R. S.; MOTTA, T. B. N.; DANTAS, J. L. L. Correlações genéticas e análise de trilha para número de frutos comerciais por planta em mamoeiro. Pesquisa Agropecuária Brasileira, Brasília, v. 45, n. 8, p. 855-862, 2010.

OLIVEIRA, M.S.P.; FARIAS NETO, J.T. Seleção massal em açaiziero para a produção de frutos. Revista de Ciências Agrárias, Belém, n. 49, p. 145-156, 2008.

PALUDO, G.F.; SILVA, J.Z.; REIS, M.S. Estimativas de Produção de Frutos de Palmiteiro (Euterpe edulis Mart.) a partir da Densidade de Indivíduos. Biodiversidade Brasileira, São Paulo, v.2, n.2, p.92-102, 2012.

PINTAUD, J.; GALEANO, G.; BALSLEV, H.; BERNAL, R.; BORCHSENIUS, F.; FERREIRA, E.; GRANVILLE, J.; MEJÍA, K.; MILLÁN, B.; MORAES, M.; NOBLICK, L.; STAUFFER, F. W.; KAHN, F. Las palmeras de América del Sur: diversidad, distribución e historia evolutiva.

RESENDE, M. D. V. de. Software SELEGENREML/BLUP: sistema estatístico e seleção genética computadorizada via modelos lineares mistos. Colombo: EMBRAPA Florestas, 2007. 359 p.

SANTOS, V. S.; NARDINI, V.; CUNHA Jr, L. C.; BARBOSA Jr, F.; TEIXEIRA, G. H. A. Identification of species of the Euterpe genus by rare earth elements using inductively coupled plasma mass spectrometry and linear discriminant analysis. Food Chemistry, Berlin, v. 153, p. 334-339, 2014.
SGROTT, A. F.; BOOZ, M. R.; PESCADOR, R.; HECK, T. C.; STURMER, S. L. Arbuscular mycorrhizal inoculation increases biomass of euterpe edulis and archontophoenix alexandrae after two years under field conditions. Revista Brasileira de Ciências do Solo, Viçosa, MG, v. 36, p.1103-1112, 2012.

SINDHUMOLE, P.; IBRAHIM, K. K. Path analysis of oil yield in coconut (Cocos nucifera L.). Geobios, Jodhpur, v. 29, n. 4, p. 239-240, 2002.

SHIMIZU, J. Y. Estratégia complementar para conservação de espécies florestais nativas: resgate e conservação de ecótipos ameaçados. Pesquisa Florestal Brasileira, Colombo, n.54, p.07-35. 2007.

TEIXEIRA, D. H. L.; OLIVEIRA, M. S. P.; GONCALVES, F. M. A.; NUNES, J. A. R. Correlações genéticas e análise de trilha para componentes de produção de frutos de açaizeiro. Revista Brasileira de Fruticultura, Jaboticabal, v. 34, n. 4, p. 1135-1142, 2012.

TEAM, R. C. A language and environment for statistical computing. Vienna: R Foundation for Statistical Computing, 2013. Disponível em: $<$ http:// www.R-project.org/>. Acesso em: 9 fev. 2014.

VENCOVSKY, R.; BARRIGA, P. Genética biométrica no fitomelhoramento. Ribeirão Preto: Sociedade Brasileira de Genética, 1992. 496p.

WRIGHT, S. Correlation and causation. Journal of Agricultural Research, Washington, v. 20, p. 557-585, 1921

ZAMBRANA, N. Y. P.; BYG, A.; SVENNING, J. C.; MORAES, M.; GRANDEZ, C.; BALSLEV, H. Diversity of palm uses in the western Amazon. Biodiversity Conservation, Andova, v.16, p.27712787, 2007. 